\title{
Generation of Arbitrary Quadrature-Amplitude Modulated Signals Using a Single Dual-Drive Modulator
}

\author{
Keang-Po Ho \\ Institute of Communication Engineering and Dept. of Electrical Engineering \\ National Taiwan University, Taipei 106, Taiwan \\ E-mail: kpho@cc.ee.ntu.edu.tw
}

\begin{abstract}
Regardless of complexity, all types of quadrature-amplitude modulated (QAM) signal can be generated using a single dual-drive Mach-Zehnder modulator. Three different quadrature phase-shift keying (QPSK) transmitters are proposed as examples.
\end{abstract}

\section{Introduction}

Recently, multilevel modulation schemes like differential quadrature phase-shift keying (DQPSK) have received renewed attention [1]-[4]. Not only for DQPSK or QPSK, but applicable to general class of quadratureamplitude modulation (QAM) with and without differential operation, Fig. 1(a) is the traditional method to generate a QAM signal using two Mach-Zehnder modulators (MZMs) within two $\pi / 2$-phase difference paths of an Mach-Zehnder interferometer. Intuitively, the two MZMs generate the signal for the two quadrature components with a phase difference of $\pi / 2$, i.e., $\cos (\cdot)$ and $\sin (\cdot)$. As shown later, QAM signal can also be generated using a single dual-drive MZM as shown in Fig. 1(b).

\section{General Principles}

If the two paths of a dual-drive MZM have independent driving voltages of $V_{1}$ and $V_{2}$, with an input electric field of $E_{i}$, the output electric field is

$$
E_{o}=\frac{E_{i}}{2}\left[\exp \left(j \pi \frac{V_{1}}{V_{\pi}}\right)+\exp \left(j \pi \frac{V_{2}}{V_{\pi}}\right)\right],
$$

where $V_{\pi}$ is the voltage to provide a $\pi$ phase shift of each phase modulator. In additional to operate a MZM

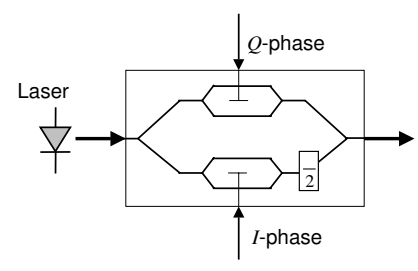

(a)

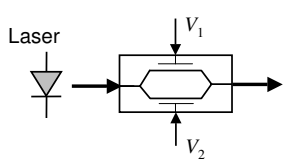

(b)
Fig. 1. (a) The traditional QAM transmitter based on two MZMs in an interferometer. (b) A QAM transmitter based on a single dual-drive MZM. as a phase modulator with $V_{1}=V_{2}$, by choosing both $V_{1}$ and $V_{2}$ of (1) properly, any quadrature signal can be generated. Assumed an $M$-ary signal constellation that can be represented as complex numbers of

$$
s_{i}=r_{i} e^{j \theta_{i}}, \quad i=0, \ldots, M-1,
$$

with a maximum amplitude of $r_{\max }=$ $\max \left\{r_{0}, r_{1}, \ldots, r_{M-1}\right\}$. The input and output relationship of (1) is rewritten in the following normalized form of

$$
E_{o}=\frac{r_{\max }}{2}\left(e^{j \phi_{1}}-e^{j \phi_{2}}\right),
$$

where $\phi_{1}=\pi V_{1} / V_{\pi}$ and $\phi_{2}=\pi V_{2} / V_{\pi}+\pi$. The output electric field of $E_{o}$ is the difference of two vectors in the circle having a radius of $r_{\max } / 2$. The MZM of (3) is biased at the minimum transmission point or the null point and the maximum output electric field has an amplitude of $r_{\max }$ when $V_{1}=V_{2}$.

With two phases of [5], [6]

$$
\begin{aligned}
\phi_{i 1} & =\theta_{i}+\cos ^{-1}\left(r_{i} / r_{\max }\right) \\
\phi_{i 2} & =\theta_{i}-\cos ^{-1}\left(r_{i} / r_{\max }\right)+\pi,
\end{aligned}
$$

we obtain

$$
s_{i}=\frac{r_{m}}{2}\left(e^{j \phi_{i 1}}-e^{j \phi_{i 2}}\right),
$$

because of $e^{j \cos ^{-1} r_{i} / r_{\max }}+e^{-j \cos ^{-1} r_{i} / r_{\max }}=$ $2 r_{i} / r_{\max }$. All constellation points of (2) can be generated based on two phase modulators having the phases of (4) and (5), respectively.

Figs. 2 show a 16-QAM constellation and the phases of (4) and (5) for the dual-drive MZM. Instead of representing all 16 constellation points in the same figure, Fig. 2(a) decomposes the 16-QAM signal into two QPSK and one 8-PSK signals. Figs. 2(b)-(d) are the corresponding $\phi_{i 1}$ and $\phi_{i 2}$ of all 16 points according to the decomposition of Fig. 2(a). The empty and solid circles correspond to $\phi_{i 1}$ and $\phi_{i 2}$, respectively. Fig. 2(b) gives the QPSK signal of Fig. 2(a) with maximum amplitude of $r_{\max }$. The two closest points of Fig. 2(b) are the same but draw differently for the phases of two 


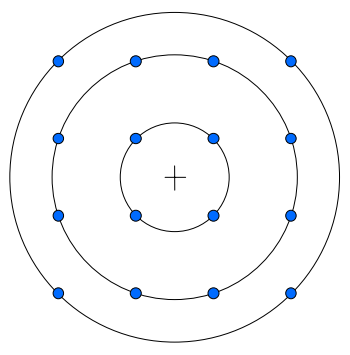

(a) 16-QAM

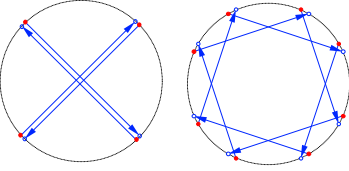

(b) Big QPSK

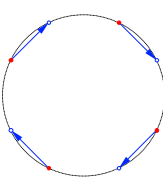

(d) Small QPSK
Fig. 2. (a) 16-QAM constellation and its decomposition into two QPSK and an 8-PSK signals, (b), (c), (d) are the two phases of $\phi_{1}$ (empty circles) and $\phi_{2}$ (solid circles) to generate the 16-QAM signals.

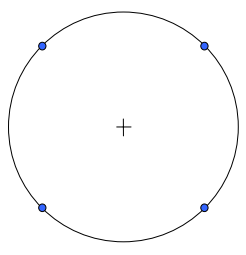

(a) QPSK

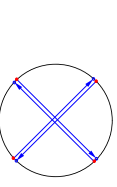

(b) 4 level

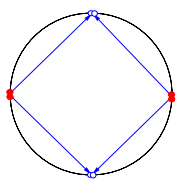

(c) 2 level (c) 8 PSK

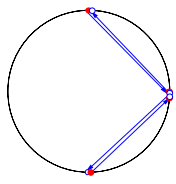

(d) 3 level
Fig. 3. (a) QPSK constellation and its generation using a (b) four-, (c) two-, and (d) three-level drive signal.

different signals. Fig. 2(c) generates the 8-PSK signal of Fig. 2(a). Fig. 2(d) gives the QPSK signal of Fig. 2(a) with the smallest amplitude.

From Fig. 2, the generation of a 16-QAM signal using a dual-drive MZM requires the usage of a 16-level drive voltage and thus very complicated drive circuits. Compared with the transmitter of Fig. 1(a), the simplification of the optical components using a single dualdrive MZM creates a high complexity in the electronic circuits. With the allowance of higher modulator loss, as shown later, the number of drive levels can be reduced.

\section{Generation of (D)QPSK Signals}

The dual-drive MZM transmitter of Fig. 1(b) can use to generate either QPSK or DQPSK signals with a constellation of Fig. 3(a). Similar to that of Fig. 2(b), Fig. 3(b) is the trivial case of operating the dual-drive MZM as a phase modulator with $V_{1}=V_{2}$. The four phases of Fig. 3(a) is given by a four-level drive signal. A QPSK signal may be generated with higher loss but smaller number of levels if the four constellation points are generated with phases of $\phi_{1}$ or $\phi_{2}$ the same as one another. With only two values of $\phi_{1}$ and $\phi_{2}$, Fig. 3(c) drives the dual-drive MZM in Fig. 1(b) with a two-level signal. Fig. 3(d) has three different values of $\phi_{1}$ and $\phi_{2}$ and requires a three-level drive signal. Comparing Figs. 3(c) and (d) with Fig. 3(b), the schemes using two and three level drive signal have $3-\mathrm{dB}$ extra loss than that of

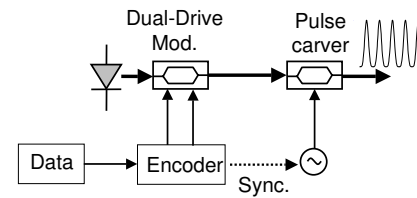

Fig. 4. A RZ-DQPSK transmitter

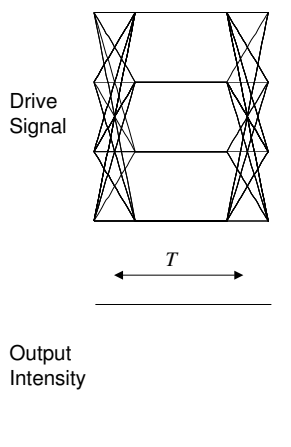

(a)

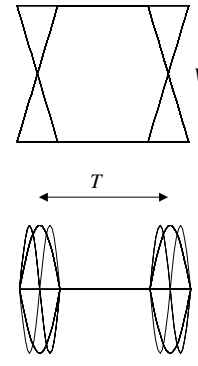

(b)
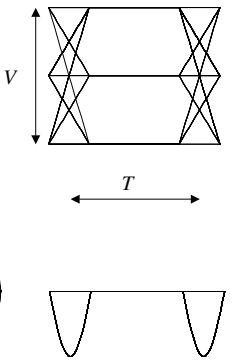

(c)
Fig. 5. The eye-diagram of the drive signal and output intensity between two modulators of the transmitter of Fig. 4 when (a) 4, (b) 3 , (c) 2 drive signals are used.

Fig. 3(b). Both the peak-to-peak drive voltage and the number of drive levels are reduced in Figs. 3(c) and (d).

However, both the two- and three-level transmitters of Figs. 3(b) and (c) do not have constant intensity between consecutive symbols. Fig. 4 shows a RZDQPSK transmitter using a dual-drive MZM followed by a phase craver. After the phase craver, the intensity is a constant pulse train. However, Figs. 5 show the eyediagram of the drive signal and the intensity before the phase craver. With two or three levels of drive signal, the output intensity of the dual-drive MZM has some ripples between consecutive symbols. The simplest twolevel scheme of Fig. 3(c) and 5(b) has a peaking ripple doubling the output intensity. The two- and three-level drive signals of Fig. 3(c) and (d) are applicable for RZ but not NRZ signals. The peak-to-peak drive voltage is reduced from $1.5 V_{\pi}$ for four-level signal to $V_{\pi}$ for twoand three-level drive signals.

\section{Conclusion}

Using 16-QAM and QPSK signals as example, we show that a single dual-drive MZM can be used to generate all types of QAM signals.

\section{References}

[1] R. A. Griffin et al. OFC '02, paper FD6.

[2] P. S. Cho et al. IEEE Photon. Technol. Lett. 15, 473 (2003).

[3] H. Kim and R.-J. Essiambre, IEEE Photon. Technol. Lett. 15, 769 (2003).

[4] N. Yoshikane and I. Morita, $O F C$ '04, paper PDP38.

[5] D. C. Cox, IEEE Trans. Commun. 22, 1942 (1974).

[6] D. C. Cox and R. P. Leck, IEEE Trans. Commun. 23, 1281 (1975). 